\title{
Hétérogénéité spatiale d'une forêt tropicale humide de Sumatra : effet de la topographie sur la structure floristique
}

\author{
V Trichon \\ Laboratoire d'écologie terrestre, UMR 5552 (CNRS / UPS), 13, avenue du Colonel-Roche. \\ BP 4403, 31405 Toulouse cedex, France"
}

(Reçu le ler avril 1996; accepté le 3 juin 1996)

\begin{abstract}
Summary - Spatial heterogeneity of a tropical rain forest in Sumatra: effect of topography on floristic structure. Tropical rain forests are characterized by a complex and spatially heterogeneous structure. It is essential to analyze the factors of this heterogeneity in order to set up sustainable management policies in these environments. We have studied the influence of topography on floristic structure, considering 3 ha of forest with contrasted relief. Correspondance analysis has shown that topography acts through drainage, leading to different specific compositions on ridge, slope and valley. It operates also in relation to forest dynamics, favouring pioneer specics on steep slopes. In addition, this influence decreases as topographic heterogeneity is reduced; other factors related to forest internal characteristics then govern floristic structure. Moreover, this study brings new data concerning floristic composition and ecology of forests in this area, as well as topographical preferences of many species. Topography appears to be a key factor regarding the structure and the dynamics of tropical rain forests on contrasted relief. and should be considered in modelling.
\end{abstract}

tropical rain forest / spatial heterogeneity / floristic structure / topography / Sumatra

Résumé - Les forêts tropicales humides se caractérisent par une structure complexe très hétérogène dans l'espace. L'étude des facteurs responsables de cette hétérogénéité est indispensable si l'on veut mettre en place des aménagements durables dans ces milieux. À Sumatra, sur une parcelle forestière de 3 ha au relief contrasté, nous avons étudié l'influence de la topographic sur l'hétérogénéité spatiale de la structure floristiçue. Les résultats d'une analyse factorielle des correspondances montrent que la topographie intervient par le biais du drainage. en induisant une composition spécifique différente entre vallée, pente et crête. Elle intervient aussi en relation avec le cycle sylvigénétique, en favorisant la présence d'espèces pionnièress sur de fortes pentes. L'analyse montre encore que cette influence s'estompe lorsque le niveau d hétérogénéité du terrain diminue, et que d'autres facteurs liés aux particularités internes à la forêt (dynamique naturelle, diversité spécifiçue...) régissent alors la struc-

\footnotetext{
* Laboratoire d’accueil en Indonésie : Scameo-Biotrop. PO Box 17, Bogor. Indonésie.
} 
ture floristique. Cette étude apporte des données nouvelles sur la composition floristique et l'écologie des forêts de cette région, ainsi que sur les préférences topographiques de nombreuses espèces d'arbres. La topographie apparaît comme un facteur clé de la structure et de la dynamique des forêts tropicales humides sur terrain accidenté, et elle devrait être prise en compte dans leur modélisation.

forêt tropicale humide / hétérogénéité spatiale / structure floristique / topographie / Sumatra

\section{INTRODUCTION}

Botanistes et phytogéographes reconnaissent depuis longtemps la nature hétérogène et morcelée des forêts tropicales humides (Richards, 1952 ; Whitmore, 1984).

La complexité de la structure forestière que l'on observe à l'échelle d'une station (structure floristique et tridimensionnelle) provient notamment du grand nombre des espèces végétales, de leur architecture variée et de la coexistence de différentes phases de développement à un instant donné.

L'hétérogénéité structurale de ces forêts et leur richesse spécifique élevée sont souvent interprétées en termes de dynamique forestière et en relation avec les phénomènes de succession qui en résultent (Aubréville, 1938 ; Budowski, 1965 ; Hallé et al, 1978 ; Denslow, 1980, 1987).

D'autres études mettent l'accent sur le rôle des variations locales de la topographie (Kahn. 1983 ; Basnet, 1992 ; Tuomisto et al, 1995) ou des paramètres édaphiques (Ashton, 1964 ; Newbery et Proctor, 1984 ; Lescure et Boulet, 1985 ; Gartlan et al, 1986; Steege et al, 1993).

Les caractéristiques biologiques des espèces (reproduction, régénération, dissémination...) influencent aussi leur distribution spatiale. Ces particularités sont le plus souvent inconnues, ce qui complique encore notre compréhension de l'hétérogénéité forestière.

Enfin, d'autres paramètres interviennent de manière difficilement évaluable, tels que le vent ou les animaux qui participent à la dissémination des graines (Sabatier, 1983 ; Charles-Dominique, 1995).
L'étude des facteurs qui influencent l'hétérogénéité spatiale de la structure forestière (dynamique naturelle, nature topographique ou édaphique du terrain, biologie des espèces, agents disséminateurs) est fondamentale pour notre connaissance de ces milieux et nécessaire, de ce fait, à toute décision concernant leur aménagement ou leur conservation.

À l'heure actuelle, les forêts de plaine de l'île de Sumatra, en Indonésie, n'existent plus que sous une forme très dégradée (Laumonier, 1991). Les forêts de basse colline, entre 200 et $500 \mathrm{~m}$ d'altitude, sont à leur tour de plus en plus menacées par l'exploitation forestière, l'agriculture itinérante sur brûlis ou l'établissement de plantations industrielles. C'est à ces altitudes que nous avons mené nos recherches, dans une région de l'île où règne une forte pression pour l'accès aux terres forestières. Nous nous sommes intéressés particulièrement à l'influence de variations locales de la topographie sur la structure floristique, à travers l'étude de la distribution spatiale des espèces d'arbres forestiers.

\section{MATÉRIEL ET MÉTHODE}

\section{Site d'étude}

Le site de recherche est localisé à une latitude de $1^{\circ} 37^{\circ} \mathrm{S}$ et une longitude de $101^{\circ} 47^{\prime} \mathrm{E}$. Le climat présente deux saisons marquées, une saison des pluies d'octobre à avril et une saison moins humide de mai à septembre. Les précipitations annuelles sont comprises entre 2500 et $3000 \mathrm{~mm}$ et la température moyenne mensuelle varie entre 25 et $27^{\circ} \mathrm{C}$ (Fontanel et Chantefort, 1978). 
La station d'étude, établie sur terrain granitique, correspond à 3 ha $(100 \mathrm{~m} \times 300 \mathrm{~m})$ de forêt naturelle non perturbée par l'activité humaine. C'est un transect comprenant une zone de vallée située à proximité d'une importante rivière, et le terrain accidenté des collines adjacentes. L'altitude varie entre 150 et $230 \mathrm{~m}$, et la station est divisée pour les besoins de l'analyse en trois parcelles de 1 ha, chacune divisée en quadrats de $20 \mathrm{~m}$ par $20 \mathrm{~m}$ (fig 1). La méthode d'échantillonnage et de mise en place des parcelles dérive de celle préconisée par Brünig et Synnot (1977) pour des sites d'étude permanents en forêt dense humide. Elle est similaire aux méthodes d'inventaires forestiers utilisées pour établir la typologie de stations en régions tempérées, à la différence que, en raison d'une richesse spécifique beaucoup plus importante, l'étude est ici limitée aux espèces ligneuses. Les distances ont été mesurées en projection horizontale à l'aide d'un mètre à ruban et d'un clinomètre, qui permet également de relever régulièrement la topographie. Chaque arbre de plus de $10 \mathrm{~cm}$ de diamètre a été numéroté, repéré par ses coordonnées et récoltél. Les identifications ont été faites par comparaison aux nombreux spécimens de l'herbier du Biotrop ${ }^{2}$, où les échantillons ont été déposés ainsi qu'à l'Herbier de Montpellier. Au total, 1874 arbres ont été recensés (soit une densité moyenne de 624,7 arbres/ha), répartis en 57 familles et 471 espèces.

\section{Méthode}

Pour mettre en évidence les phénomènes de variation de la composition floristique sur cette station, en relation avec les variations des caractéristiques topographiques, nous avons d'abord utilisé l'analyse factorielle des correspondances (AFC) du logiciel ADE (Chessel et Dolédec, 1994). Pour préciser les résultats de l'AFC concernant les préférences topographiques de certaines espèces, nous avons ensuite étudié indépendamment la répartition spatiale de quelques-

1. Les récoltes ont été réalisées avec l'aide d'un macaque (Macaca nemestrina) entrainé à cette tâche par un dresseur d'un village voisin. 2. Laboratoire de biologie tropicale de la Seameo (Organisation des ministères de l'Éducation des palys d'Asie du Sud-Est), situé à Bogor, île de Java.

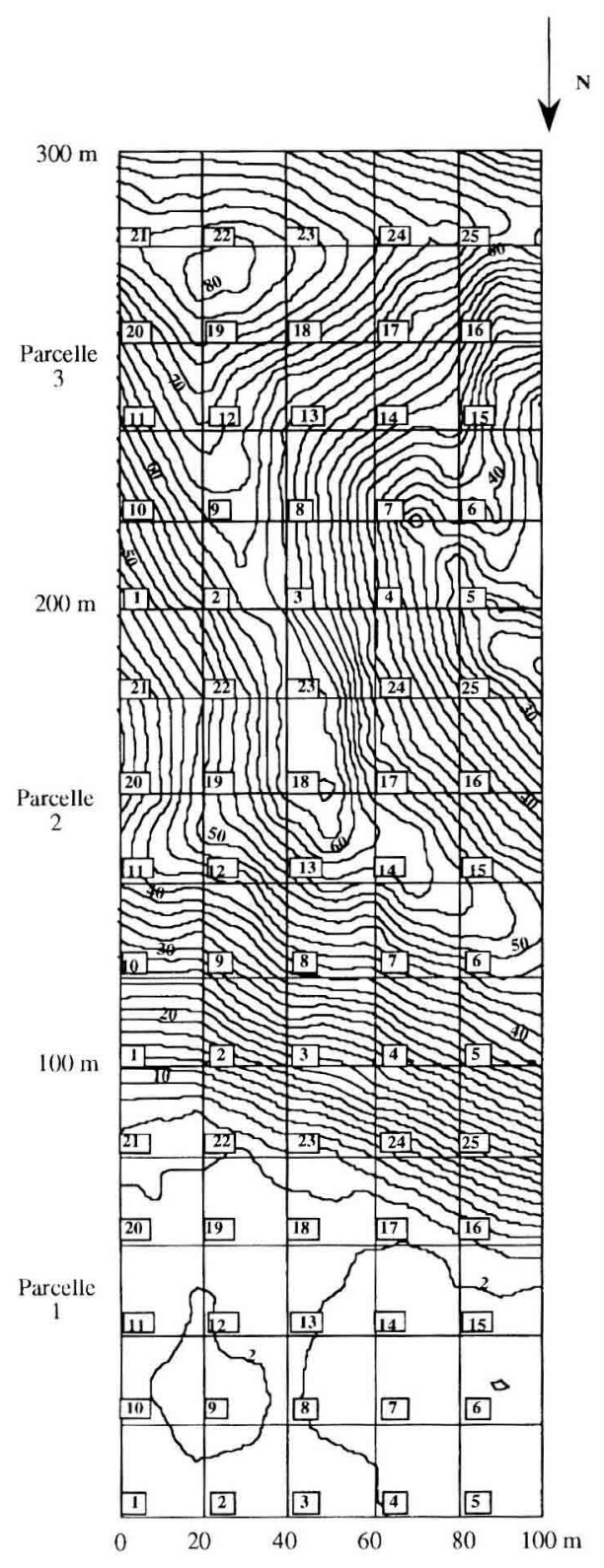

Fig 1. Topographie et partition de la station d'étude. Dénivelé : $150 \mathrm{~m}-230 \mathrm{~m}$; numérotation des quadrats : de 1 à 25 pour chacune des parcelles de 1 ha :

: courbes de niveau relatives tous les $2 \mathrm{~m}$. 
unes d'entre elles, parmi les plus abondantes, en tenant compte de leur effectif réel.

Les modalités choisies pour la réalisation de l'AFC sont les suivantes.

L'unité statistique d'échantillonnage est le quadrat de $20 \mathrm{~m}$ par $20 \mathrm{~m}$, parce qu'il correspond bien à la taille des unités de la mosaïque forestière, ou «éco-unités » au sens d'Oldeman (1990). Des quadrats de taille inférieure $(10 \mathrm{~m} \times 10 \mathrm{~m})$ présenteraient moins de risque d'être hétérogènes d'un point de vue topographique, mais seraient faiblement représentés à cette limite de diamètre (six individus par quadrat en moyenne). Excepté au niveau le plus étroit de la crête, l'unité statistique d'échantillonnage choisie reste cohérente avec la maille d'hétérogénéité topographique sur la station.

L'utilisation du critère de présence ou d'absence des espèces a apporté de mcilleurs regroupements que la prise en compte de leur abondance. Ce critère permet également de limiter les effets du grégarisme (dû à une dissémination barochore ou à des rejets naturels), qui pourrait masquer l'influence des facteurs environnementaux sur la structure floristique (Austin et Greig-Smith, 1968).

En raison de la grande diversité spécifique, l'analyse a été allégée par l'élimination d'un certain nombre d'espèces rares, qui risqueraient de brouiller les résultats sans contribuer à la structure floristique d'ensemble. Cette sélection permet aussi d'éliminer l'effet des coapparitions peu significatives entre espèces rares (Austin et Greig-Smith, 1968). Le seuillage adopté ici, limité aux espèces apparaissant dans plus de quatre quadrats, a éliminé environ $79 \%$ du nombre total d'espèces recensées sur la parcelle et $56 \%$ du nombre total d'individus. Cela revient finalement à conduire une $\mathrm{AFC}$ avec 98 espèces représentées par 826 individus, répartis dans 75 quadrats.

\section{RÉSULTATS}

\section{Analyse factorielle des correspondances}

\section{Résultats bruts de l'AFC}

Il est question dans cette étude des trois premiers facteurs de l'AFC (valeurs propres de $0,69,0,36$ et 0,32 ). L'interprétation des groupements de points sur les axes se fait en fonction des variables (quadrats ou espèces) de plus fortes contributions absolues et relatives (Jambu, 1989).

Une analyse détaillée des deux premiers facteurs est présentée ici, illustrée avec la projection des points quadrats (fig 2 ). L'axe 1 permet d'individualiser trois groupes de quadrats que nous avons appelés $\mathrm{A}, \mathrm{B}$ et $\mathrm{C}$. Chaque groupe correspond à une classe de coordonnées, que nous avons subdivisée en deux dans le cas du groupe $\mathrm{C}$, en raison du grand nombre des points et pour les besoins de l'interprétation. Sur l'axe 2, trois groupes de quadrats se distinguent aussi (D, E et $F$ ) qui correspondent à trois classes de coordonnées.

Les résultats obtenus sur l'axe 3 de l'AFC sont plus difficiles à interpréter en raison d'une répartition diffuse des quadrats de contribution significative. Ces résultats seront néanmoins présentés car ils apportent des éléments de discussion intéressants.

\section{Interprétation de l'axe I}

La figure 3 sent de référence aux discussions suivantes. Elle permet de visualiser la correspondance entre les groupes de quadrats définis pour l'axe 1 et les caractéristiques topographiques du terrain associé. On y a également indiqué la présence des espèces de plus fortes contributions, ou qui sont importantes pour la compréhension de la structure floristique.

Les quadrats du groupe A à l'extrémité positive de l'axe ( $\mathrm{n}^{\circ} 1.1$ à 1.23 ) correspondent au terrain plat de la vallée. La distinction floristique très nette de l'ensemble de ces quadrats nous permet d'ores et déjà de définir les limites géographiques de la forêt ripicole. La grande majorité d'entre eux se situent à moins de $4 \mathrm{~m}$ au-dessus du niveau de la rivière.

Les quadrats du groupe $C$ en position négative sur l'axe sont situés sur les pentes et les crêtes de la station, à une élevation de plus de $40 \mathrm{~m}$ par rapport à la rivière. 


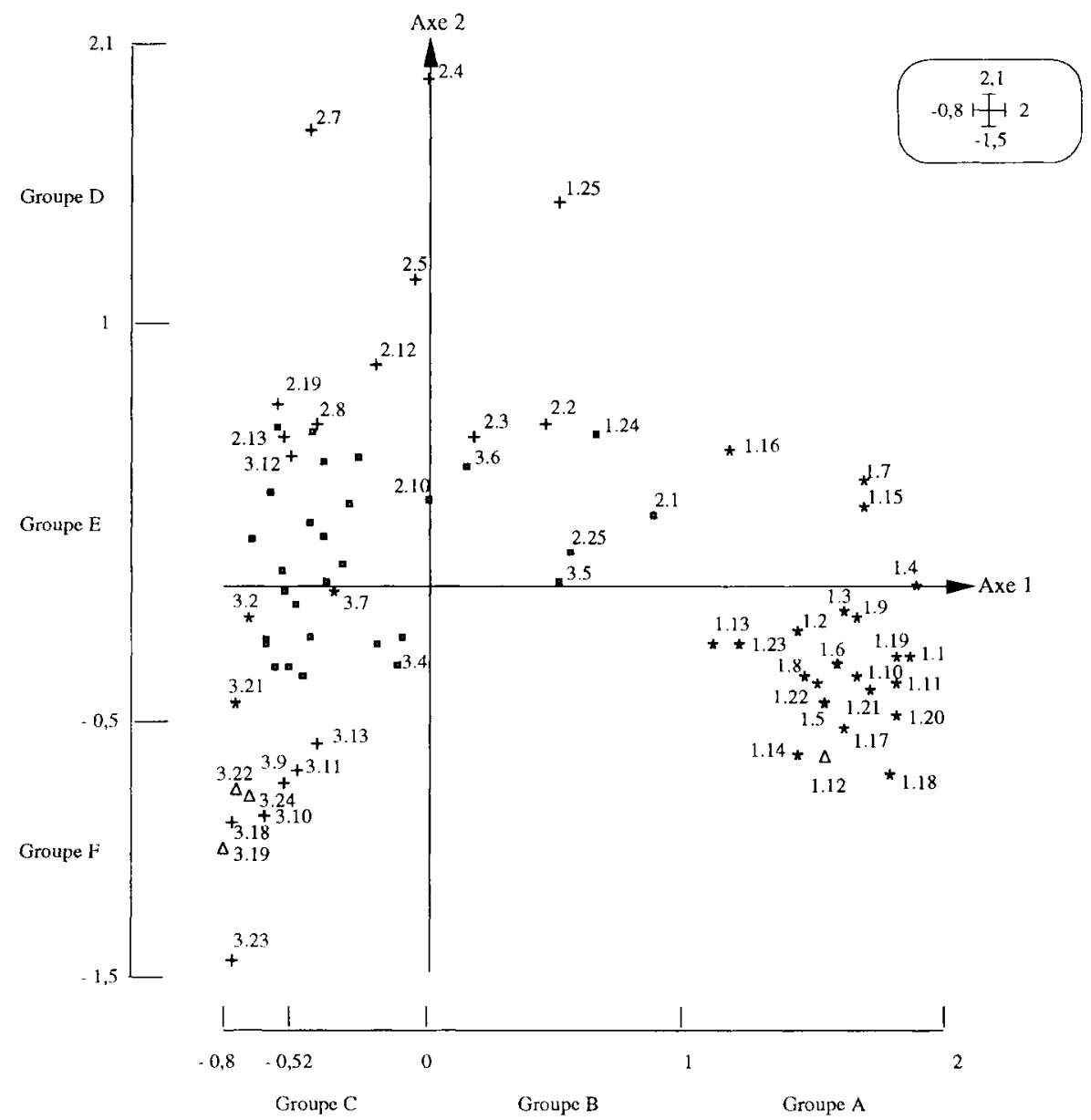

Fig 2. Projection des quadrats sur le plan des axes 1 et 2 de l'AFC. * : quadrats de fortes contributions sur l'axe $1 ;+:$ quadrats de fortes contributions sur l'axe $2: \Delta:$ quadrats de fortes contributions sur les deux axes; $\mathbf{a}:$ autres quadrats.

Au sein du groupe B, cinq quadrats ont sur la parcelle une position intermédiaire entre ceux des groupes A et C (n ${ }^{\text {os }} 1.24$, $1.25,2.1,2.2$ et 2.3 ). Ce caractère intermédiaire concerne à la fois leur localisation géographique et leur altitude (ce sont des pentes situées à moins de $40 \mathrm{~m}$ au-dessus de la rivière). Les trois autres quadrats du groupe B sont situés dans les collines mais incluent un vallon étroit parcouru par un ruisseau ( $n^{\circ} 2.25$ et 3.5$)$ ou bordent ce vallon $\left(n^{\circ} 3.6\right)$.

La localisation sur la parcelle des cinq quadrats du groupe B situés entre la vallée et les collines explique que s"y retrouvent ensemble des espèces caractéristiques des deux biotopes, d'où leur position intermédiaire sur l'axe. Cette même explication ne convient pas cependant pour les trois autres 


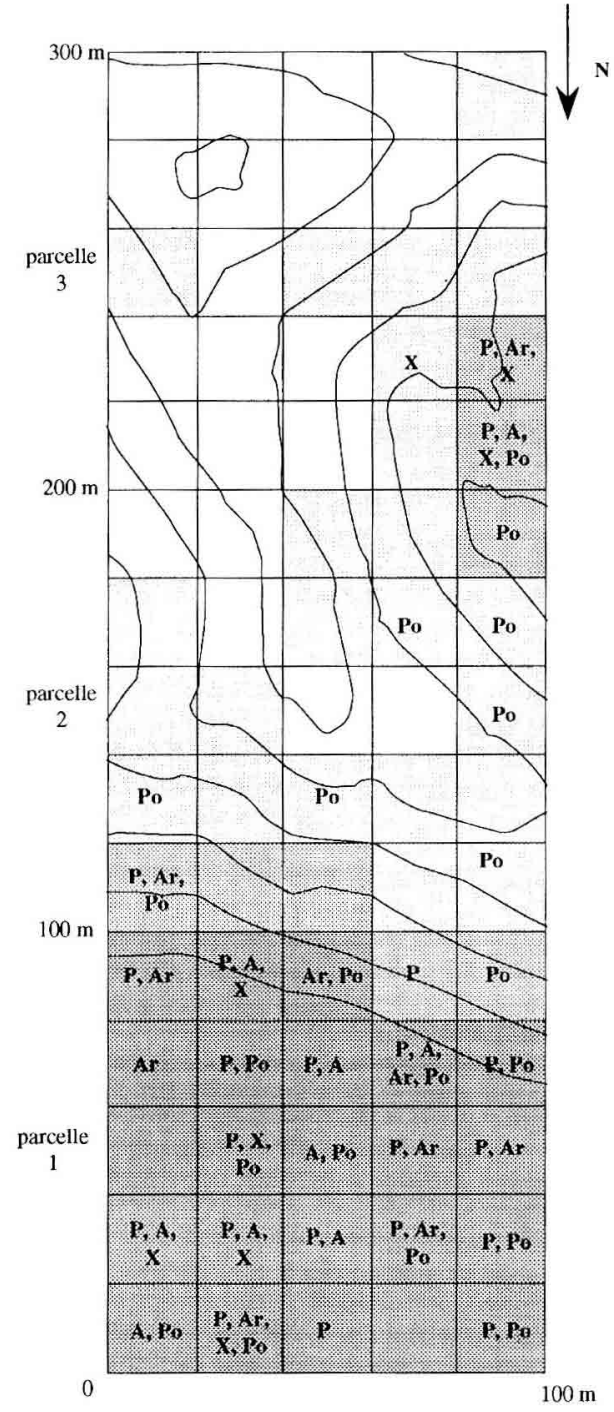

Fig 3. Répartition spatiale des groupes de quadrats sur l'axe 1 et des espèces importantes pour l'interprétation de l'axe.

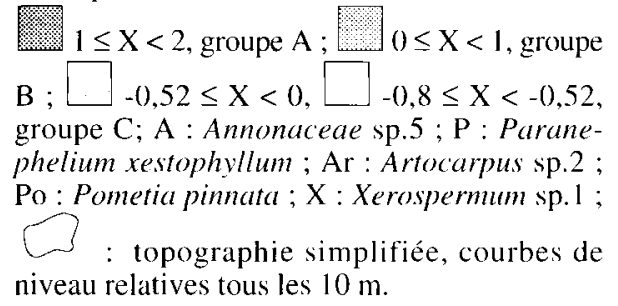

quadrats du même groupe, où l'on retrouve les espèces Annonaceae sp 5 (Annonaceae), Artocarpus sp 2 (Moraceae), Paranephelium xestophyllum, Pometia pinnata et Xerospermum sp 1 (Sapindaceae), toutes caractéristiques du groupe A. De fait, ces espèces retrouvent dans le vallon une humidité du sol comparable à celle de la vallée, fréquemment inondée en saison des pluies.

Un caractère commun aux quadrats du groupe C est, au contraire, qu'ils sont confinés à des terrains bien drainés, de haut de pente ou de sommet, où la disponibilité en eau dans le sol est moindre.

On peut donc considérer que la répartition des espèces et des quadrats sur l'axe l est liée dans ses grandes lignes à un gradient d'humidité du sol, lui-même imposé par la configuration topographique de la parcelle.

Le grand nombre de points du groupe $\mathrm{C}$ incite secondairement à y rechercher une sous-structure. À la suite de notre précédente interprétation concernant l'influence de la topographie sur la composition floristique, liée à un facteur de disponibilité en eau dans le sol, nous pouvons attendre l'existence d'une différenciation au sein du groupe $C$ entre les quadrats de pentes et de crêtes. Cette distinction n'apparaît pas sur la représentation graphique de l'AFC (fig 2), parce qu'elle constitue un phénomène mineur par rapport au regroupement des quadrats de la vallée et à l'individualisation des groupes A, B et C sur l'axe 1 . On peut voir cependant sur la figure 3 que l'agencement des quadrats de la quatrième classe de coordonnées suit une courbe globalement assimilable à la ligne de crête principale, les imperfections pouvant être expliquées par la topographic mixte de certains quadrats.

L'effet d'un gradient d'humidité du sol sur la structure floristique, mis en évidence sur la base d'un contraste marqué de composition entre vallée et collines, persiste donc à un niveau plus fin de variation topographique entre les pentes d'une part et les 
crêtes d'autre part. Ces dernières présentent en effet une disponibilité en eau dans le sol plus éphémère encore.

\section{Interprétation de l'axe 2}

La répartition spatiale des trois groupes de quadrats sur l'axe 2, ainsi que la distribution des espèces de plus forte contribution sur ce même axe, sont présentées sur la figure 4. Les quadrats du groupe $\mathrm{A}$, qui se sont distingués sur l'axe l comme étant floristiquement très différents des autres et qui ne participent pas de manière significative à la construction de l'axe 2, n'ont pas été pris en compte dans cette figure.

Les quadrats du groupe $\mathrm{D}$, isolés à l'extrémité positive de l'axe, sont caractérisés principalement par des Euphorbiaceae (Trigonopleura malayana et Macaranga motleyana, mais aussi $M$ hosei et $M$ gigantea) ainsi que par une Rubiaceae (Porterandia anisophylla). À l'exception de T malayana, rarement mentionnée pour ses préférences écologiques dans la littérature, ces espèces sont connues pour être des pionnières, caractéristiques de la phase de régénération en forêt primaire après perturbation naturelle. Elles apparaissent ensemble sur une zone de fortes pentes avoisinant $60^{\circ}$

Fig 4. Répartition spatiale des groupes de quadrats sur l'axe 2 et des espèces importantes pour l'interprétation de l'axe.

$1 \leq X<2,1$, groupe $D$; $-0,5 \leq X<1$, groupe $\mathrm{E} ; \square-1,5 \leq \mathrm{X}<-0,5$, groupe $\mathrm{F}$; $\square$ quadrats non pris en compte; coordonnées négatives : K : Kokoona reflexa, D : Drypetes sp.2, P : Polyalthia hypoleuca, Ho: Horsfieldia polvspherula, Hy : Hydnocarpus kunstleri, $\mathrm{T}$ : Teijsmanniodendron coriaceum ; coordonnées positives: M : Macaranga motleyana, $\mathrm{Tr}:$ Trigonopleura malayana, $\mathrm{Mg}$ : Macaranga gigantea, Mh : Macaranga hosei, $\mathrm{Pa}:$ Porterandia anisophylla; $\square$ : topographie simplifiée, courbes de niveau relatives tous les $10 \mathrm{~m}$. où, par ailleurs, l'absence d'individus de gros diamètre témoigne d'un cycle sylvigénétique court (Trichon, 1996). Les signes d'érosion intense observés à cet endroit du terrain renforcent l'hypothèse d'un milieu peu propice à l'établissement d'un couvert forestier stable. Ainsi le rassemblement de ces espèces sur plusieurs quadrats proches est représentatif d'un terrain particulièrement exposé aux perturbations naturelles.

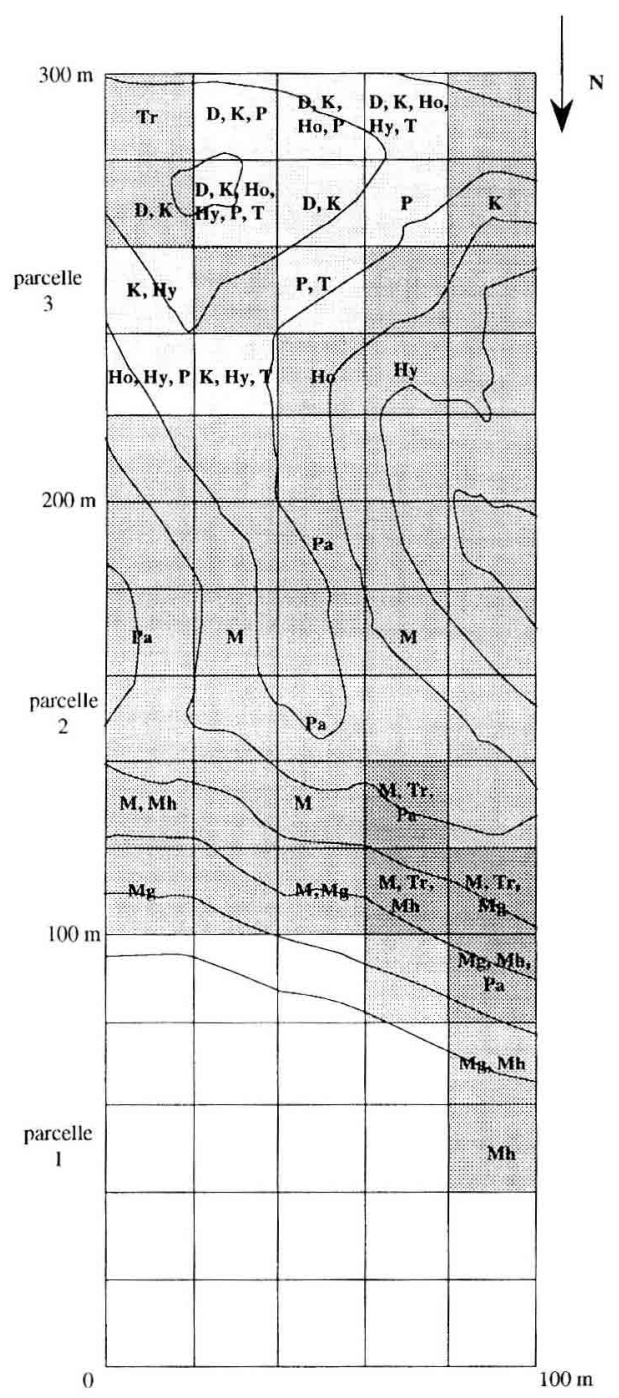


Les quadrats du groupe $\mathrm{F}$, à l'extrémité négative de l'axe 2, sont eux aussi presque contigus mais correspondent à un facies topographique différent, de crête ou de haut de pente. Les espèces qui les caractérisent sont pour certaines limitées aux étages inférieurs de la canopée, comme Polyalthia hypoleuca (Annonaceae), Horsfieldia polyspherula (Myristicaceae), Hydnocarpus kunstleri (Flacourtiaceae) ou Drypetes sp 2 (Euphorbiaceae). D'autres ont des statures intermédiaires comme Teijsmanniodendron coriaceum (Verbenaceae), ou constituent la strate supérieure ou émergente telle Kokoona

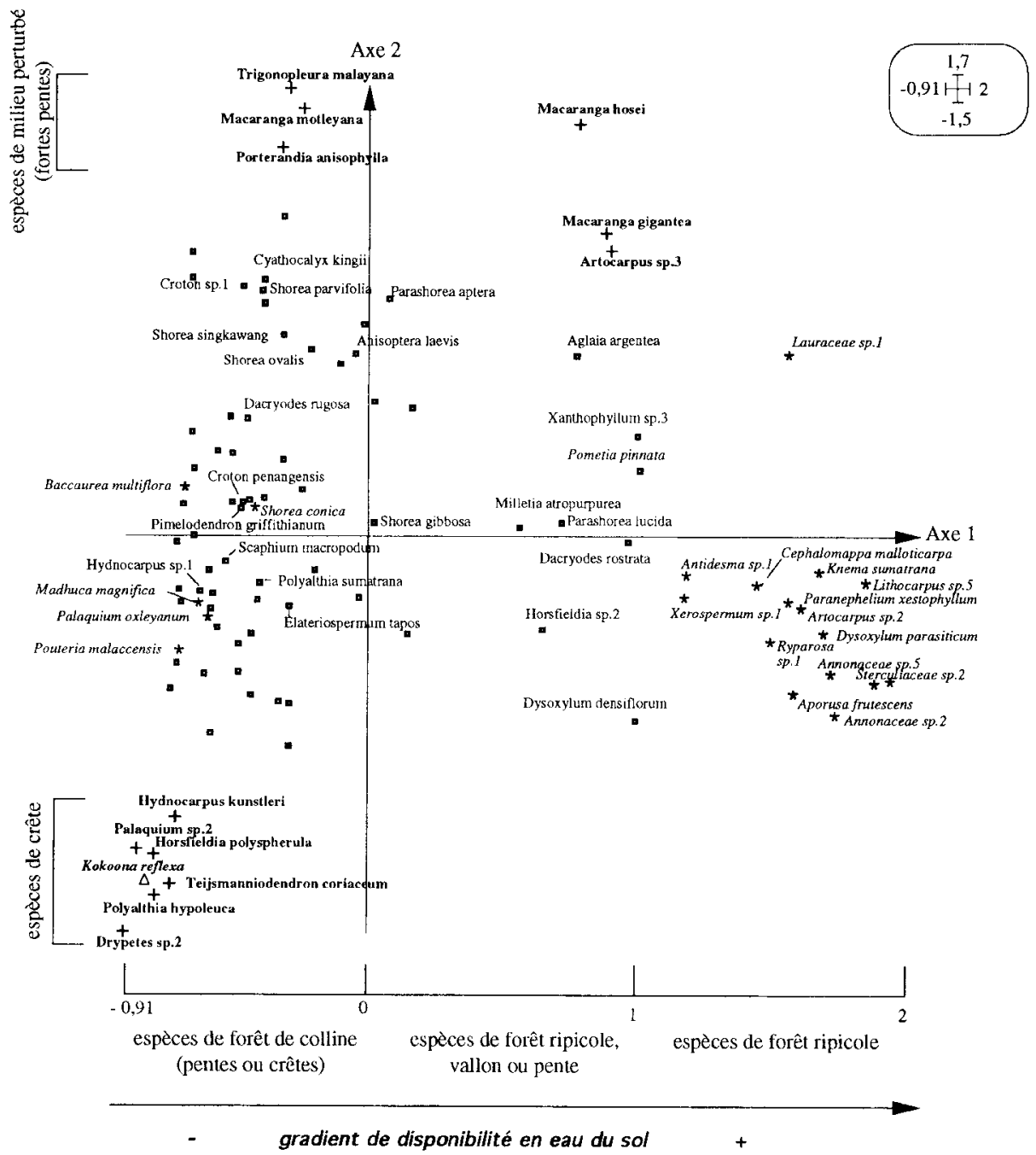

Fig 5. Interprétation de la projection des espèces sur le plan des axes 1 et 2 de l'AFC. * nom en italique : espèces de fortes contributions sur l'axe $1 ;+$ nom en gras : espèces de fortes contributions sur l'axe $2 ; \triangle$ nom en italique et gras : espèces de fortes contributions sur les deux axes; $\square$ nom en romain : autres espèces considérées pour l'interprétation des axes. 
reflexa (Celastraceae), décrite comme un grand arbre des forêts de crêtes à Bornéo (Kochummen et Whitmore, 1972). Ces espèces, recensées dans différentes stations forestières de cette région de Sumatra (Trichon, 1996), ont toujours été associées à une phase sylvigénétique mature. Dans cc secteur de la parcelle où l'on ne détecte pas de traces de perturbations naturelles actuelles ou antérieures, la forêt présente une phase sylvigénétique plus ancienne et un cycle de régénération plus long que sur les pentes du précédent groupe.

La structure floristique principale mise en évidence par l'axe 2 est donc une opposition entre deux secteurs forestiers de facies topographique distinct, subissant un degré différent de perturbations naturelles et présentant un stade différent de maturité.

La figure 5 récapitule l'interprétation des deux premiers axes de l'analyse en présentant la projection des points espèces. Les noms de certaines espèces de faibles contributions n'ont pas été reportés afïn d'alléger la figure. On retrouve la même structure de groupes de points que pour la projection des quadrats. L'analyse de ces deux axes permet ainsi d'identifier les préférences écologiques d'un grand nombre d'espèces.

\section{Interprétation de l'axe 3}

Les quadrats qui ont eu des contributions significatives sur l'axe 3 sont limités au groupe A et présentent une répartition diffuse. De la même façon, l'observation de la projection des espèces n'a pas permis de définir de groupements nets.

Cela nous amène à la conclusion que les modalités de l'AFC que nous avons choisies ne sont plus adaptées quand il s'agit de mettre en évidence une structure spatiale sur une surface d'environ 1 ha ne présentant pas d'hétérogénéité marquée du terrain. Le découpage de la parcelle en quadrats de $20 \mathrm{~m}$ par $20 \mathrm{~m}$ constitue en effet une approximation trop grossière par rapport à la petite surface concernée. Les informations portant uniquement sur les espèces de plus de $10 \mathrm{~cm}$ de diamètre deviennent insuffisantes, d'autant plus que la densité en forêt ripicole est relativement faible (411 arbres/ha).

\section{Répartition spatiale précise de quelques espèces}

L'influence de la topographic sur la composition floristique a été mise en évidence sur les deux premiers axes de l'AFC. La préférence écologique pour un facies de crête ou de pente est cependant restée indéterminée pour les espèces correspondant à l'intersection des groupes $\mathrm{C}$ et $\mathrm{E}$. Afin de compléter cette information, la répartition spatiale précise de certaines espèces a été analysée en tenant compte de leur effectif exact. Nous n'avons étudié que les espèces représentées par plus de dix individus, considérant cette abondance comme un minimum pour rendre compte de leur préférence topographique.

Nous illustrons cette étude avec la famille des Dipterocarpaceae, abondamment représentée sur notre parcelle en forêt de colline. La figure 6 met en évidence la variabilité des préférences topographiques que peuvent présenter différentes espèces de cette famille. Il apparaît clairement que Shorea conica, très abondante sur la parcelle, montre une occurrence nettement supérieure sur les crêtes. $S$ parvifolia et Parashorea aptera sont essentiellement localisées sur les hauts de pente. $P$ lucida semble s'adapter, au contraire, à toutes les situations topographiques, avec une préférence pour les bas de pente.

D'autres exemples ont permis d'illustrer différentes tendances de répartition des espèces en fonction de la topographie.

Une Euphorbiaceae, Baccaurea multiflora, et trois Sapotaceae, Madhuca magnifica, Palaquium oxleyanum et Pouteria 


\section{$\downarrow^{N}$}

$300 \mathrm{~m}$

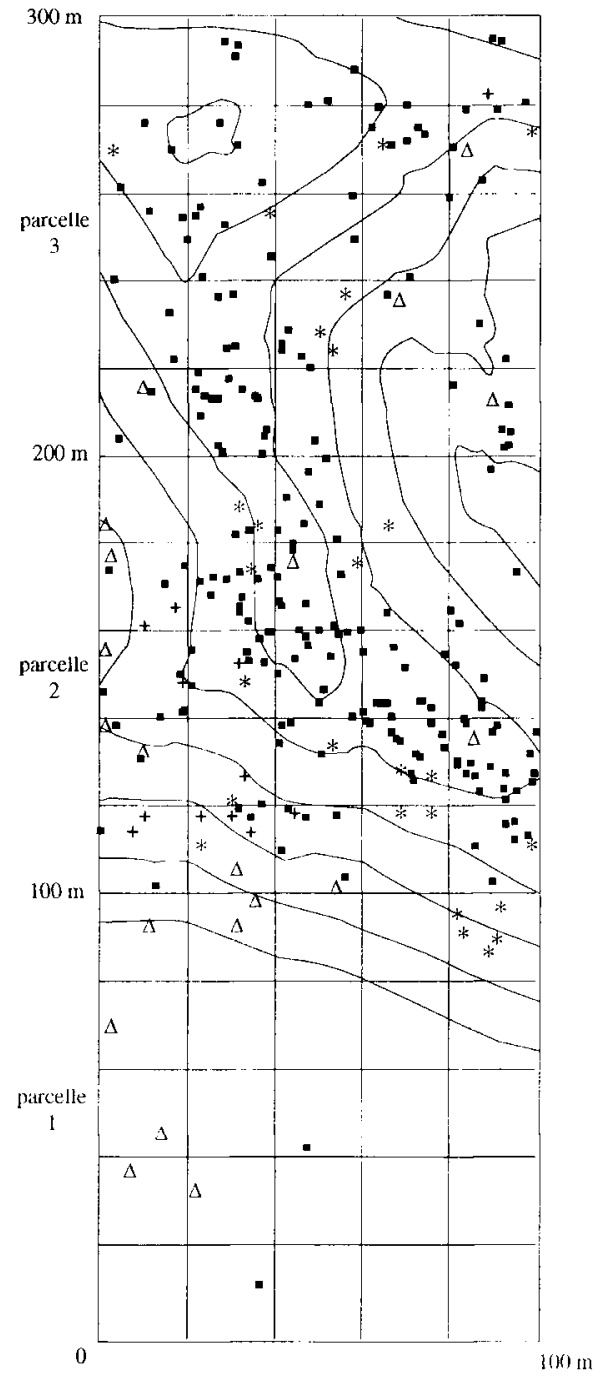

Fig 6. Distribution de quelques espèces de Dipterocarpaceae selon la topographic. Effectifs selon les espèces : $\mathbf{0}$ : Shorea conica (194);*: Shorea parvifolia (21); $\triangle$ : Parashorea lucida (13) ; +: Parashorea aptera (12).

Z : topographie simplifiée, courbes de niveau relatives tous les $10 \mathrm{~m}$. malaciensis, ont montré une répartition nette le long de la ligne de crête.

Trois Euphorbiaceae, Croton penangensis, Elateriospermum tapos et Pimelodendron griffithianum, ont présenté une nette préférence pour les pentes.

Enfin, pour d'autres espèces telles que Crathocalyx kingii et Polyalthia sumatrana (Annonaceae), Dacryodes rugosa (Burseraceae) ou Hydrocarpus sp 1 (Flacourtiaceae), la répartition a semblé indifférente à la topographie.

La figure 7 récapitule les préférences écologiques de l'ensemble des espèces considérées dans cette étude, préférences mises en évidence à travers les résultats de l'analyse factorielle, d'une part, et grâce à ceux des observations complémentaires ci-dessus, d'autre part.

\section{DISCUSSION}

La structure floristique de la parcelle forestière apparaît fortement liée aux caractéristiques topographiques du site à travers deux phénomènes distincts. La topographie intervient sur la distribution spatiale des espèces en déterminant, d'une part, des zones écologiquement différentes par leur caractéristiques générales de drainage. Ce sont principalement la vallée et les collines qui marquent cette distinction floristique, les espèces considérées étant pour la plupart limitées à l'un ou l'autre de ces deux biotopes. La topographie intervient, d'autre part, en influant sur la dynamique forestière au niveau de zones de fortes pentes.

\section{Forêt ripicole}

Les tableaux I et II présentent les valeurs d'abondance et de dominance des principales familles de forêt ripicole sur la parcelle. La particularité de ce type de forêt est depuis longtemps mentionnée dans la littérature (Richards, 1952) et reconnue par les 


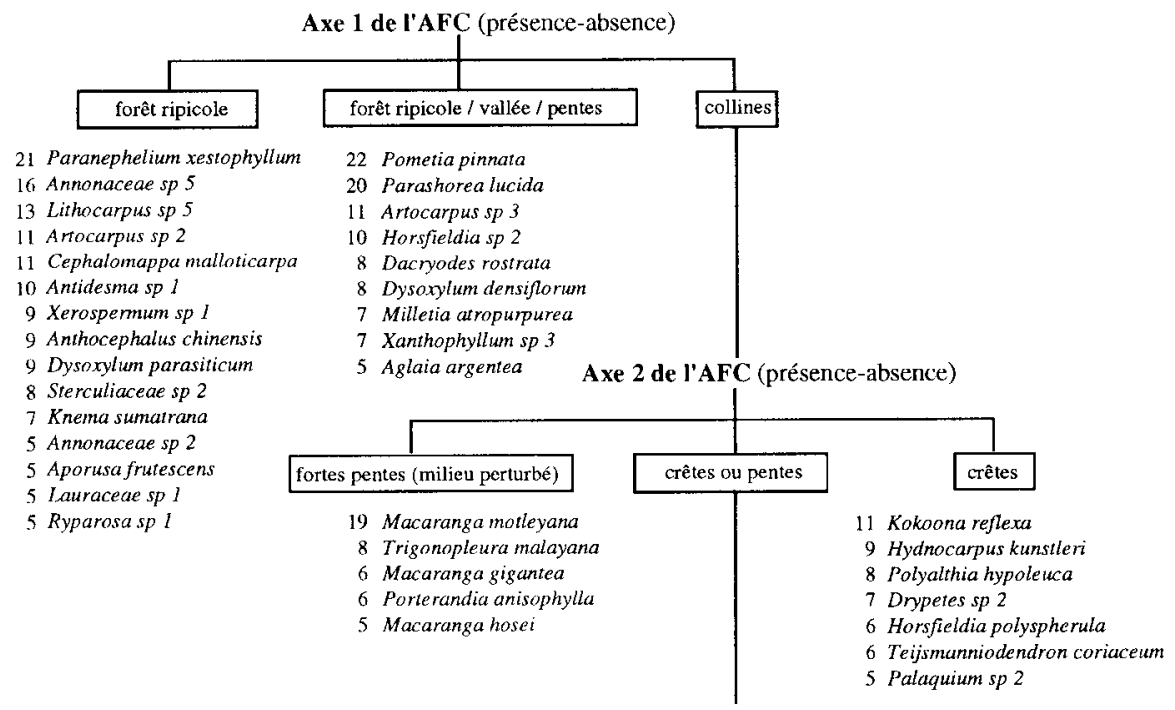

Observations complémentaires (abondance)

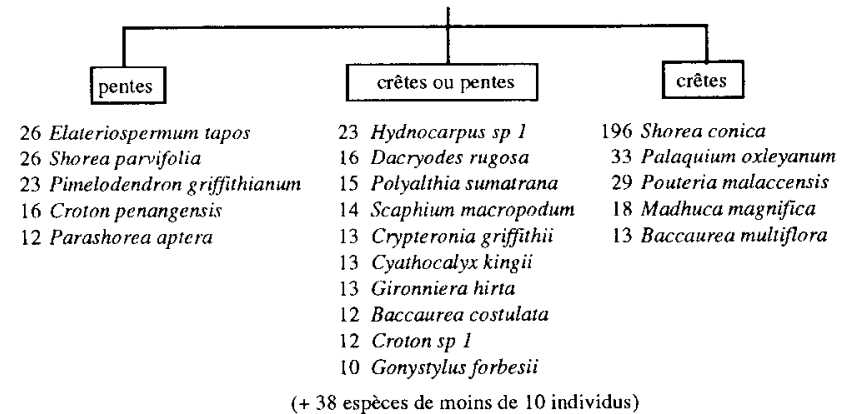

Fig 7. Préférence écologique des espèces ordonnées par abondance décroissante.

Tableau I. Liste des dix familles de plus forte abondance en forêt ripicole.

\begin{tabular}{lccr}
\hline Famille & $\begin{array}{c}\text { Effectif } \\
(1 \text { ha })\end{array}$ & $\begin{array}{c}\text { Abond } \\
\text { rel(\%) }\end{array}$ & $\begin{array}{c}\text { Nb. } \\
\text { espèces }\end{array}$ \\
\hline Sapindaceae & 57 & 13,87 & 5 \\
Euphorbiaceae & 53 & 12,90 & 25 \\
Meliaceae & 48 & 11,68 & 18 \\
Annonaceae & 29 & 7,06 & 9 \\
Moraceae & 19 & 4,62 & 5 \\
Dipterocarpaceae & 18 & 4,38 & 7 \\
Sterculiaceae & 18 & 4,38 & 7 \\
Fagaceae & 17 & 4,14 & 6 \\
Lauraceae & 17 & 4,14 & 10 \\
Myristicaceae & 16 & 3,89 & 6 \\
\hline
\end{tabular}

Tableau II. Liste des dix familles de plus forte dominance en forêt ripicole.

\begin{tabular}{lcc}
\hline Famille & $\begin{array}{c}\text { Dominance } \\
\left(\mathrm{m}^{2} / \mathrm{ha}\right)\end{array}$ & $\begin{array}{c}\text { Dom } \\
\text { rel }(\%)\end{array}$ \\
\hline Bombacaceae & 4,09 & 13,55 \\
Sapindaceae & 3,26 & 10,82 \\
Rubiaceae & 2,59 & 8,58 \\
Moraceae & 2,52 & 8,35 \\
Dipterocarpaceae & 2,37 & 7,86 \\
Meliaceae & 2,33 & 7,73 \\
Euphorbiaceae & 2,25 & 7,44 \\
Sterculiaceae & 1,88 & 6,23 \\
Fagaceae & 1,65 & 5,45 \\
Lauraceae & 1,28 & 4,24 \\
\hline
\end{tabular}


cartographes. Abdulhadi et al (1991) ont étudié une forêt ripicole au nord de Sumatra et l'ont caractérisée par une forte abondance de Meliaceae, ce que nous confirmons ici. En prenant en compte la totalité des espèces recensées sur la parcelle, cette famille présente $11,7 \%$ de l'abondance totale dans la vallée, contre $2,2 \%$ dans les collines. Sa diversité spécifique importante ( 18 espèces sur 48 individus) contribue également à faire des Meliaceae une famille caractéristique de cette forêt riveraine. La plus forte abondance relative dans la vallée est cependant celle des Sapindaceae, elles aussi beaucoup mieux représentées qu'en forêt de colline $(13,9 \%$ contre $2,4 \%)$. Mais la diversité spécifique de cette famille est beaucoup plus faible avec seulement cinq espèces, dont une (Paranephelium xestophyllum) très abondante. D'autre part l'espèce Pometia pinnata, bien que connue pour être fréquente sur les berges de rivières (Whitmore, 1984), n'est pas rare en terrain mieux drainé, comme l'ont montré cette étude et de précédents résultats (Steenis, 1957 ; Laumonier, 1991). En termes de dominance, la surface terrière réunie par les Sapindaceae est importante ( $P$ pinnata peut atteindre de gros diamètres), tandis que celle des Meliaceae reste plus faible. Cinq individus seulement confèrent à la famille des Bombacaceae la plus forte dominance dans ce type forestier. Un Durio zibethinus, plus gros arbre de la parcelle avec un diamètre de $170 \mathrm{~cm}$, représente à lui seul plus de la moitié de la surface terrière de cette famille.

\section{Forêt de colline}

Les tableaux III et IV rassemblent les familles les plus abondantes et de plus forte dominance en forêt de colline. La forte abondance relative $(19 \%)$ et la très nette dominance relative $(29,3 \%)$ des Dipterocarpaceae dans ce type forestier nous permettent de l'assimiler aux forêts mixtes à Dipterocarpaceae (mixed dipterocarp rain forest),
Tableau III. Liste des dix familles de plus forte abondance en forêt de colline.

\begin{tabular}{lccc}
\hline Famille & $\begin{array}{c}\text { Effectif } \\
(2 \mathrm{ha})\end{array}$ & $\begin{array}{c}\text { Abond } \\
\text { rel(\%) }\end{array}$ & $\begin{array}{c}\text { Nb } \\
\text { espèces }\end{array}$ \\
\hline Dipterocarpaceae & 278 & 19,00 & 13 \\
Euphorbiaceae & 223 & 15,24 & 47 \\
Sapotaceae & 109 & 7,45 & 13 \\
Burseraceae & 77 & 5,26 & 17 \\
yristicaceae & 69 & 4,72 & 22 \\
Annonaceae & 62 & 4,24 & 19 \\
Flacourtiaceae & 58 & 3,96 & 7 \\
Myrtaceae & 43 & 2,94 & 20 \\
Fabaceae & 39 & 2,67 & 12 \\
Lauraceae & 39 & 2,67 & 21 \\
\hline
\end{tabular}

Tableau IV. Liste des dix familles de plus forte dominance en forêt de colline.

\begin{tabular}{lcc}
\hline Famille & $\begin{array}{c}\text { Dominance } \\
\left(\mathrm{m}^{2} / \mathrm{ha}\right)\end{array}$ & $\begin{array}{c}\text { Dom } \\
\text { rel }(\%)\end{array}$ \\
\hline Dipterocarpaceae & 10,25 & 29,26 \\
Euphorbiaceae & 3,79 & 10,81 \\
Sapotaceae & 2,26 & 6,45 \\
Burseraceae & 2,04 & 5,81 \\
Fagaceae & 1,39 & 3,95 \\
Myrtaceae & 1,25 & 3,56 \\
Myristicaceae & 1,12 & 3,20 \\
Sterculiaceae & 0,99 & 2,82 \\
Fabaceae & 0,91 & 2,60 \\
Moraceae & 0,91 & 2,59 \\
\hline
\end{tabular}

caractéristiques des formations de basse altitude en Asie du Sud-Est (Ashton, 1964, 1982 ; Whitmore, 1984).

La préférence de la plupart des espèces de Dipterocarpaceae pour les milieux bien drainés de crêtes et de hauts de pentes, mise en évidence sur la figure 6, est connue. Whitmore (1984) signale, par exemple, les fortes colonisations de Shorea curtisii sur les crêtes en Malaisie péninsulaire, qu'il relie effectivement à un facteur de drainage du sol. Les 
données floristiques résultant de travaux réalisés à Sumatra en forêt de basse colline sont cependant rares et, à notre connaissance, aucun résultat concernant l'importante faculté de colonisation de $S$ conica sur les crêtes n'a été publié.

Parmi les autres espèces de colline dont la préférence topographique a été étudiée de façon précise, on peut mentionner Elatteriospermum tapos, décrite effectivement par Ho et al (1987) comme une espèce abondante sur pentes bien drainées, pouvant supporter des stress hydriques passagers.

Au vu des tableaux I, II, III et IV, les différences de composition floristique entre forêt de colline et forêt ripicole, même au niveau taxonomique général de la famille, sont flagrantes. Seules les Euphorbiaceae, connues pour réunir un grand nombre d'espèces au tempérament et aux préférences écologiques variés, gardent une abondance relative élevée dans les deux types forestiers. La différence de structure floristique entre l'un et l'autre de ces types se traduit également par une richesse spécifique beaucoup plus faible dans la vallée (165 espèces à l'hectare pour la limite diamétrale de $10 \mathrm{~cm}$ ) par comparaison aux collines $(260$ espèces à l'hectare).

\section{Topographie et dynamique forestière}

Un groupement d'espèces pionnières est apparu sur une zone de fortes pentes, en rapport avec la brièveté du cycle sylvigénétique. Oldeman (1974) constate aussi en forêt tropicale humide une fréquence accrue des chablis sur les pentes, où il décrit le phénomène «d'imbrication forestière » entre différents ensembles structuraux. Au niveau de ces zones d'imbrication, l'augmentation de la rugosité de la canopée provoque notamment une plus grande prise au vent. Dans la région d'étude à Sumatra, le vent, bien que généralement faible, peut se déchaîner subitement sous forme de violentes rafales (Laumonier, 1991). Le phénomène de chutes à répétition est en outre amplifié sur les pentes en raison du déséquilibre initial des arbres et de leur plus grande inertie lors d'une chute. La luminosité reçue latéralement par les étages inférieurs de la forêt y est plus importante, et favorise une croissance plus rapide des végétaux ainsi que le développement d'espèces pionnières. Enfin, une étude précédente portant sur la structure tridimensionnelle de la forêt sur cette parcelle de 3 ha a montré la pauvreté en arbres de gros diamètre sur ces quadrats de forte pente (Trichon, 1996). L'ensemble de nos résultats et de ces remarques conforte donc la conclusion de Kahn (1983) selon laquelle une topographie de forte déclivité raccourcit le cycle sylvigénétique, en limitant le développement de grands arbres.

Dans ce cas, la répartition spatiale des espèces est dictée par la dynamique forestière naturelle, elle-même influencée par la topographie.

\section{Hiérarchie des facteurs d'hétérogénéité floristique}

Les axes d'importance successive de l'AFC décrivent en quelque sorte la structure floristique de la parcelle à des échelles d'observation de plus en plus fines, qui s'accompagnent d'une homogénéisation du relief sur les quadrats considérés. L'influence de la topographie sur cette structure diminue avec le niveau d'hétérogénéité du terrain, laissant alors une part plus importante à des facteurs d'origine intrinsèque. Ces idées se retrouvent ci-dessous dans la récapitulation des principaux résultats obtenus sur les trois axes.

L'axe 1 met en évidence la structure floristique principale sur l'ensemble des 3 ha. Elle est liée à la disponibilité en eau dans le sol, elle-même dépendante des caractéristiques topographiques. Les quadrats de vallée sont ainsi opposés au terrain bien drainé des collines, lequel est secondairement différencié en facies de pentes et de crêtes. 
L'axe 2 fait apparaître la structure floristique dans les 2 ha de collines, où se dégage une opposition marquée entre deux secteurs forestiers de facies topographique distinct. Cette opposition s'explique par la fréquence des perturbations naturelles sur un terrain en pentes par rapport à un terrain de crête.

L'axe 3 détaille, quant à lui, la structure floristique dans les quadrats de la vallée, que l'axe I de l'analyse avait dans un premier temps rassemblés. Sur cette petite surface de topographie homogène, la structure reste difficile à interpréter et apparaît comme le reflet de caractéristiques internes à la forêt.

On peut donc distinguer deux groupes de facteurs influençant, à des degrés différents, l'hétérogénéité spatiale de la structure floristique sur la parcelle. Les premiers, responsables des principaux groupements sur les deux premiers axes de l'AFC, sont liés aux caractéristiques du terrain, en l'occurrence à la topographie. Ils interviennent de manière prépondérante dans des cas d'hétérogénéité marquée des conditions écologiques. Les seconds correspondent aux

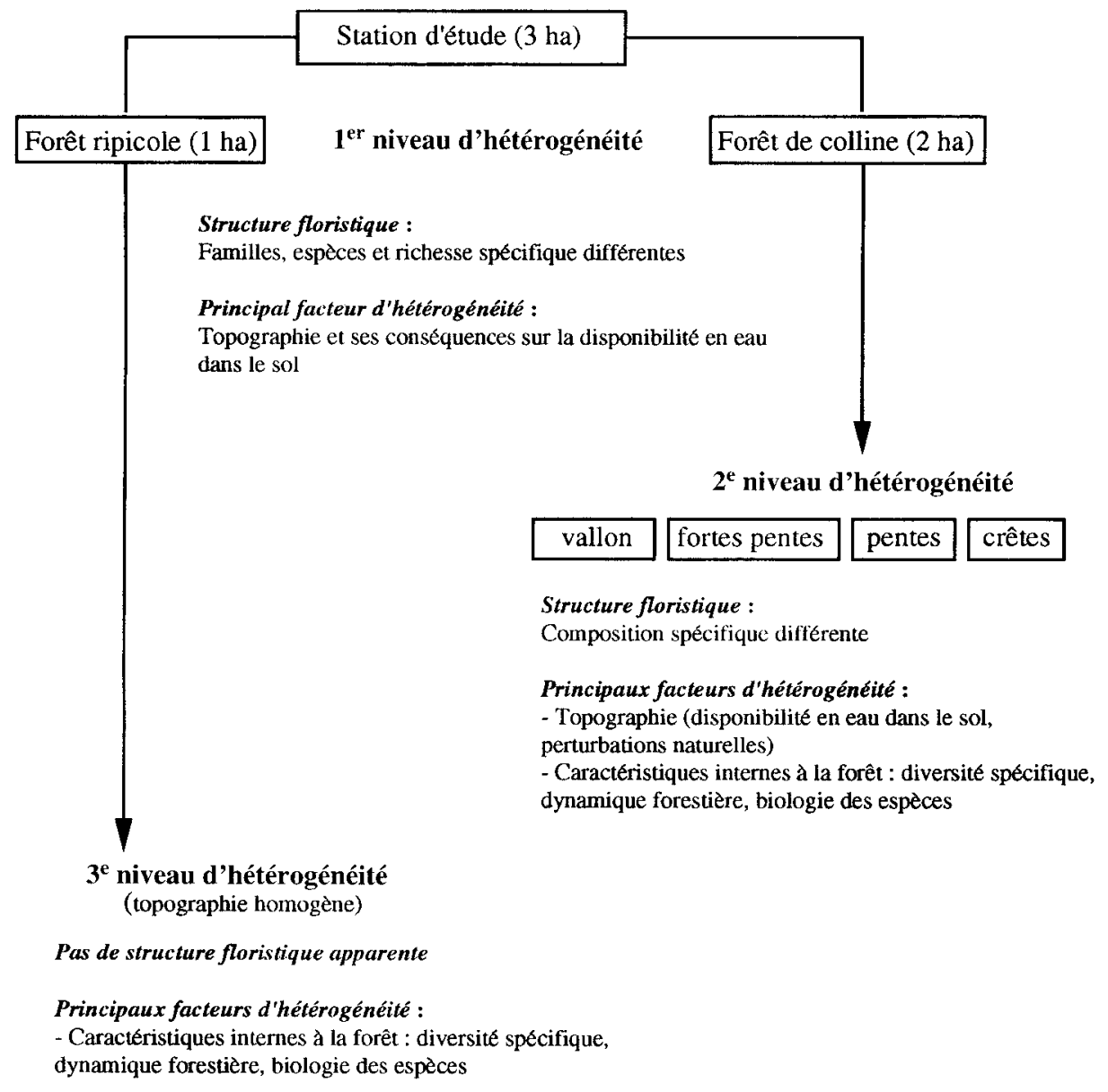

Fig 8. Hiérarchie des facteurs d'hétérogénéité floristique aux différentes échelles d'observation. 
particularités intrinsèques de la forêt : la grande diversité spécifique, les caractéristiques biologiques des espèces, la dynamique naturelle. L'influence de ce second groupe de facteurs est permanente mais est facilement supplantée par celle d'une topographie accidentée. La figure 8 illustre la hiérarchie des facteurs d'hétérogénéité forestière sur la parcelle, suivant le niveau d'hétérogénéité du terrain lui-même, à ces trois échelles d'observation.

\section{CONCLUSION ET PERSPECTIVES}

Le site d'étude choisi est particulièrement contrasté en raison de la présence de la vallée sur près du tiers de la parcelle. L'hétérogénéité topographique qu'il présente reste néanmoins représentative de ces forêts de basse colline à Sumatra. Qu'il s'agisse de terrains granitiques ou volcaniques, qui sont les deux formations géologiques les plus fréquentes de la région, le relief est partout très accentué avec des faciès de crêtes, de pentes et de vallées plus ou moins encaissées.

Ces recherches ont permis d'apporter des connaissances sur la composition floristique des forêts ripicoles, d'une part, et des forêts de basse colline, d'autre part, dans cette région de Sumatra où aucune étude de ce type n'avait encore été publiée. Des éléments concernant l'autoécologie de bon nombre d'espèces d'arbres, et en particulier leur préférence topographique, ont pu être dégagés. Les résultats dans ce domaine sont rares, et précieux pour toute décision concernant l'aménagement de ces forêts, qui sont actuellement au cour d'une politique d'aménagement global dans cette région.

Pour aider à la mise en place d'une sylviculture bien gérée en milieu tropical humide, les scientifiques se tournent de plus en plus vers la modélisation du couvert forestier (Koop, 1989 ; Favrichon, 1994 ; Houllier, 1995). Connaissances floristiques, études du comportement des espèces et recherche de groupes fonctionnels dans la dynamique globale de peuplement alimentent ces modèles, qui sont encore loin cependant de répondre aux questions posées (Pascal, 1995). Notre étude a permis de présenter la topographie du site comme un facteur prépondérant de l'hétérogénéité spatiale des structures floristiques, pouvant exercer une influence sur la dynamique forestière. $\mathrm{Ce}$ résultat, en accord avec de précédentes observations (Pelissier, 1995), souligne la nécessité de prendre en compte le facteur topographie pour une modélisation plus juste des forêts tropicales humides sur terrain accidenté.

\section{REMERCIEMENTS}

Cette étude, menée dans le cadre d'une thèse au Laboratoire d'écologie terrestre de Toulouse, a bénéficié d'accords de coopération entre l'université Paul-Sabatier (Toulouse) et le Biotrop (Bogor). Je remercie le Biotrop et son directeur M Soekotjo, ainsi que l'équipe du Programme écologie forestière dirigée par $\mathrm{M}^{\mathrm{me}} \mathrm{U}$ Syafii pour son accueil et sa collaboration sur le terrain. Mes remerciements vont aussi à l'ensemble du Laboratoire d'écologie terrestre, et particulièrement à mon directeur de thèse, Y Laumonier, pour son encadrement en Indonésie, à A Fabre pour ses conseils en analyses de données, et à J Fontes, F Fromard et $\mathrm{H}$ Puig pour leurs précieux commentaires sur cet article.

\section{RÉFÉRENCES}

Abdulhadi R, Kartawinata K, Yusuf R (1991) A riverine tropical rain forest in Ketambe, Gunung Leuser National Park, Sumatera, Indonesia. In : Fourth Round-table Conference on Dipterocarps. Biotrop, Bogor. Biotrop Special Publ 41, 247-256

Armesto JJ, Mitchell JD, Villagran C (1986) A com parison of spatial patterns of trees in some tropical and temperate forests. Biotropica 18, 1-11

Ashton PS (1964) Ecological Studies in the Mixed Dipterocarp Forests of Brunei State. Oxford Forestry Memoirs, 25. Clarendon Press, Oxford

Ashton PS (1982) Dipterocarpaceae. In : Flora Malesiana (Van Steenis, ed). Martinus Nijhoff, La Haye, Ser I 9 (2), 237-552 
Aubréville A (1938) La forêt coloniale : les forêts de l'Afrique occidentale française. Ann Acad Sci Coloniales $9,1-245$

Austin MP, Greig-Smith P (1968) The application of quantitative methods to vegetation survey. II. Some methodological problems of data from rainforest. $J$ Ecol 56, 327-344

Basnet K (1992) Effect of topography on the pattern of trees in tabonuco (Dacryodes excelsa) dominated rain forest of Puerto-Rico. Biotropica 24, $31-42$

Budowski G (1965) Distribution of tropical American rain forest species in the light of successional processes. Turrialba 15, 40-42

Brünig EF, Synnot TJ (1977) An outline for a basic interdisciplinary research programme with emphasis on a common approach to research methodology and measurement techniques. In : Trans of the Int MAB-IUFRO workshop on tropical rain forest ecosystems research. Hambourg. Vol Special Report 1, 276-342

Charles-Dominique P (1995) Interactions plantes-animaux frugivores, conséquences sur la dissémination des graines et la régénération forestière. $\operatorname{Rev} E \mathrm{Col}$ (Terre et Vie) 50, 223-235

Chessel D, Dolédec S (1994) Analyses multivariées et expression graphique des données environnementales, version 3.6. Ura CNRS 1451, université Claude-Bernard, Lyon

Denslow JS (1980) Gap partitioning among tropical rainforest trees. Biotropica $12,47-55$

Denslow JS (1987) Tropical rain forest gaps and tree species diversity. Ann Rev Ecol Syst 18, 431-45I

Favrichon V (1994) Classification des espèces arborées en groupes fonctionnels en vue de la réalisation d'un modèle dynamique de peuplement en forêt guyanaise. Rev Ecol (Terre et Vie) 49, 379-403

Fontanel J, Chantefort A (1978) Bioclimats du monde indonésien (Bioclimates of the Indonesian archipelago). Trvx Sec Sci Tech Inst Fr, Pondichéry

Gartlan JS, Newbery M, Thomas DW, Waterman PG (1986) The influence of topography and soil phosphorus on the vegetation of Korup Forest Reserve, Cameroon. Vegetatio 65, 131-148

Greig-Smith P, Austin MP, Whitmore TC (1967) The application of quantitative methods of vegetation survey. I. Association analysis and principal component ordination of rain forest. J Ecol 53, 483503

Hallé F, Oldeman RAA, Tomlinson PB (1978) Tropical Trees and Forests: An Architectural Analysis. Springer-Verlag, Berlin

Ho CC, Newbery DM, Poore MED (1987) Forest composition and inferred dynamics in Jengka Forest Reserve, Malaysia. J Trop Ecol 3, 25-56

Houllier F (1995) À propos des modèles de la dynamique des peuplements hétérogènes : structures, processus démographiques et mécanismes de régulation. Rev Ecol (Terre et Vie) 50, 273-282

Hubbell SP, Foster RB (1983) Diversity of canopy trees in a neotropical forest and implications for conservation. In : Tropical Rain Forest, Ecology and Management (SL Sutton, TC Whitmore, AC Chadwick, eds), Blackwell Sci Publ (2), 25-41

Jambu M (1989) Exploration informatique et statistique des données. Dunod, Paris

Kahn F (1983) Architecture comparée de forêts tropicales humides et dynamique de la rhizosphère. Thèse doct État, USTL Montpellier, 426 p

Kartawinata K, Abdulhadi R, Partomihardjo T (1981) Composition and structure of a lowland dipterocarp forest at Wanariset, East Kalimantan. Malay For 44, 397-406

Kochummen KM, Whitmore TC (1972) Celastraceae. In : Tree Flora of Malaya (TC Whitmore, ed). Longman, Singapour (1), 156-171

Koop H (1989) Forest Dynamics - SILVI-STAR: A Comprehensive Monitoring System. Springer-Ver lag, Berlin

Laumonier Y (1991) Végétation de Sumatra, Indonésie : écologie, flore, phytogéographie. Thèse doct État, UPS Toulouse, $337 \mathrm{p}$

Lescure JP, Boulet R (1985) Relationships between soil and vegetation in a tropical rain forest in French Guiana. Biotropica 17, 155-160

Newbery DM, Proctor J (1984) Ecological studies in four contrasting lowland rain forests in Gunung Mulu National Park, Sarawak; association between tree distribution and soil factors. $J$ Ecol 72, 475 493

Oldeman RAA (1974) L'Architecture de la forêt guyanaise. Mém Orstom, Bondy, $204 \mathrm{p}$

Oldeman RAA (1990) Forests: Elements of Silvology. Springer-Verlag, Berlin

Pascal JP (1995) Quelques exemples de problèmes posés à l'analyste et au modélisateur par la complexité de la forêt tropicale humide. Rev Ecol (Terre et Vie) 5, 237-249

Pelissier R (1995) Relations entre l'hétérogénéité spatiale et la dynamique de renouvellement d'une forêt dense humide sempervirente. Thèse doct Univ Lyon I, $236 \mathrm{p}$

Richards PW (1952) The Tropical Rain Forest. Cambridge University Press, Cambridge

Sabatier D (1983) Fructification et dissémination en forêt guyanaise. Thèse $3^{\mathrm{e}}$ cycle, USTL Montpellier, $238 \mathrm{p}$

Ter Steege H, Jetten VG, Polak AM, Werger MJA (1993) Tropical rain forest types and soil factors in a watershed in Guyana. J Veg Sci 4, 705-716

Van Steenis CGGJ (1957) Outlines of vegetation types in Indonesia and some adjacent regions. In : Proc VIII Pac Sc Congress Manila (4), 61-97

Trichon V (1996) Hétérogénéité spatiale des structures en forêt naturelle de basse altitude à Sumatra, Indonésie. Thèse Doct Univ Toulouse III, 269 p

Tuomisto H, Ruokolainen K, Kalliola R, Linna A, Danjoy W, Zoila R (1995) Dissecting Amazonian Biodiversity. Science 269, 63-66

Whitmore TC (1984) Tropical Rain Forest of the Far East. Clarendon Press, Oxford 